\title{
CRONOTIPO E QUALIDADE DO SONO DE ACADÊMICOS DO PRIMEIRO ANO DO CURSO DE MEDICINA DA CIDADE DE MARINGÁ-PR
}

\section{Bruna Jordana de Mello}

Acadêmica do curso de Medicina da Universidade Estadual de Maringá (UEM). Bolsista PIBIC-AF, Brasil.

\section{Sonia Trannin de Mello}

Doutora em Ciências Biológicas; Docente no Departamento de Ciências Morfológicas; Anatomia Humana da Universidade Estadual de Maringá (UEM), Brasil.

\section{Ana Paula Vidotti}

Doutora em Ciências; Docente no Departamento de Ciências Morfológicas; Anatomia Humana da Universidade Estadual de Maringá (UEM), Brasil.

\section{Josiane Medeiros de Mello}

Doutora em Ciências Biológicas. Docente no Departamento de Ciência Morfológicas; Anatomia Humana da Universidade Estadual de Maringá (UEM), Brasil.
RESUMO: O impacto da má qualidade do sono e da dimensão matutinidade e vespertinidade pode interferir no comportamento social e na saúde do indivíduo. Além das funções fisiológicas, o sono é fundamental para a consolidação da memória. O objetivo deste estudo foi avaliar o cronotipo e qualidade de sono dos 31 alunos do primeiro ano do curso de medicina da Universidade Estadual de Maringá, no Paraná. Foi utilizado o questionário cronobiológico, para avaliação do cronotipo do indivíduo e PSQI, que verifica a qualidade de sono. Quanto ao cronotipo, perante uma amostra de 18 acadêmicos analisados, oito deles mostraram-se com tendência à vespertinidade, seis moderadamente vespertinos e quatro indiferentes. Para a qualidade do sono, dos 31 acadêmicos, 61,29\% $(\mathrm{n}=19)$ apresentaram qualidade ruim de sono. Os resultados chamam a atenção para a necessidade de intervenções destinadas à promoção de ações preventivas e de autocuidado sobre a importância do sono para o bem-estar físico, psicológico e cognitivo.

PALAVRAS-CHAVE: Memória; Ritmo Circadiano; Estresse.

\section{CHRONOT YPE AND SLEEP QUALITY OF FIRST-YEAR UNDERGRADUATES IN THE COURSE OF MEDICINE IN MARINGÁ, BRAZIL}

\begin{abstract}
Impact by bad sleeping and morning-evening dimension may interfere in people's social behavior and health. Besides being a physiological function, sleep is basic for memory consolidation. Current study evaluates chronotype and sleep quality of 31 first-year undergraduates of the Course of Medicine at the Universidade Estadual de Maringá, Maringá PR Brazil. Chrono-biological questionnaire was employed to assess chronotype and PSQI, which verifies sleeping quality. Regard chronotype, eight out of the 18 undergraduates sampled revealed an evening trend, six were moderately evening-trended and four were neutral. In the case of sleeping quality, 19 (61.29\%) out of the 31 undergraduates had bad sleeping quality. Results show the need for intervention to promote preventive activities and self-care on the importance of sleep for the physical, psychological and cognitive wellbeing.
\end{abstract}

KEY WORDS: Memory; Circadian Rhythm; Stress.

\section{INTRODUÇÃO}

O sono, além de normalizar funções endócrinas, termorregulação, conservação e restauração de energia, tem uma 
função biológica fundamental na consolidação da memória ${ }^{1}$. É composto basicamente por duas fases distintas e alternantes, o sono de ondas lentas (NREM), em que os movimentos oculares são mínimos e o sono com movimentos rápidos dos olhos (REM). Cada uma apresenta mecanismos neurais e comportamentais distintos.

O sono NREM predomina na primeira metade da noite de sono, diminuindo gradativamente na segunda metade. Já o sono REM, torna-se maior na segunda metade da noite de sono, apresentando pico próximo ao despertar natural. Reparação celular e tecidual, liberação do hormônio do crescimento e padrão reverberativo de sinapses ocorrem durante o sono NREM; no sono REM, observamos a ocorrência de plasticidade neural e reequilíbrio afetivo ${ }^{2}$.

Durante o sono de ondas lentas, padrões de atividade neuronal ocorridos durante a vigília reverberam em várias áreas cerebrais, como hipocampo e neocórtex, com o objetivo de armazenar as informações julgadas importantes e descartar as irrelevantes, reforçando uma função biológica de grande impacto comportamental o aprendizado. No sono paradoxal (REM), a plasticidade neural promove alterações celulares estruturais transformando os traços reverberativos de memórias de curta duração em memória de longa duração $0^{3,2}$.

A compreensão desses fenômenos dinâmicos que ocorrem durante o período de sono do ciclo sono/ vigília vem evidenciando que o corpo humano trabalha em harmonia com fatores ambientais, por meio de um sistema de temporização, que atua como um marca passo, localizado no núcleo supraquiasmático do hipotálamo.

Todos os organismos multicelulares, incluindo os humanos, utilizam esse tipo de mecanismo para controlar os ritmos circadianos. Estudos recentes evidenciam que genes são regulados por esse sistema biológico e, consequentemente, um ritmo circadiano cuidadosamente calibrado adapta nossa fisiologia a diferentes fases do dia ${ }^{4}$.

Os ritmos circadianos têm um comprimento de aproximadamente 24 horas e são regulados pela luz do dia. Fatores endógenos, como, por exemplo, um déficit hormonal e ambientais como, luz, alimento, temperatura podem desalinhar os ritmos circadianos.
$\mathrm{O}$ atraso de fase fisiológico do ciclo sono-vigília, que ocorre na adolescência, associado à privação de sono, decorrente da associação de fatores pessoais, ambientais e sociais, comumente os levam à sonolência diurna, com comprometimento do desempenho escolar e prejuízos nos relacionamentos familiares e sociais ${ }^{6,7}$.

As demandas a que estão sujeitos estudantes de graduação como os do curso de medicina, que são submetidos a forte pressão e estresse pela alta carga horária do curso e pela exigência de alto rendimento, tendem a reduzir ainda mais a quantidade de sono com dessincronização do ciclo sono-vigília ${ }^{(8)}$.

Outros autores ${ }^{9)(10)(11)}$ corroboram ao afirmar que a privação do sono a que o acadêmico se submete pode levar à deterioração mental, psicológica e física, com diminuição da capacidade de raciocínio, de reter informações, de resolução de problemas e da interpretação de exames, além de se encontrarem associados a maior probabilidade de eventos adversos na saúde, com consequências econômicas e na qualidade de vida.

O objetivo do presente estudo foi avaliar os alunos do primeiro ano do curso de medicina da Universidade Estadual de Maringá, no Paraná (UEM) para verificar a prevalência de cronotipos e a qualidade de sono desses acadêmicos, com intuito de oferecer orientações que permitam entendimento das funções fisiológicas do sono e sua importância no processo de aprendizagem e memória, bem como sugerir ações que sensibilizem para a prevenção e autocuidado.

\section{METODOLOGIA}

Essa pesquisa foi desenvolvida com 31 alunos do primeiro ano do curso de medicina da Universidade Estadual de Maringá - UEM, no Paraná, no ano de 2016 (CAAE: 01220012.9.0000.0104 e Parecer aprovação n. 41853). Após apresentação dos objetivos, os e-mails dos alunos foram coletados para o envio do convite formal para participação na pesquisa.

Após, foi entregue o questionário "Pittsburgh SleepQuality Index" (PSQI) ${ }^{12}$, que avalia a qualidade de sono. O PSQI é composto por 19 itens com o propósito 
de avaliar e identificar a qualidade e distúrbios do sono nos últimos 30 dias. Os itens são utilizados como score para, subjetivamente, avaliar a qualidade do sono, a latência, a duração, a sensação de sono suficiente, os distúrbios, uso de medicamentos e a sonolência diurna.

Os escores dos sete componentes são somados para conferir uma pontuação global, que varia de $0 \mathrm{a}$ 21. Pontuações de $0-4$ indicam boa qualidade do sono, de 5-10 indicam qualidade ruim e acima de 10 indicam distúrbio do sono. Quanto maior o valor da pontuação, pior será a qualidade do sono. Um score de PSQI $>5$ indica que o indivíduo apresenta grande dificuldade em pelo menos dois componentes, ou dificuldade moderada em mais de três componentes ${ }^{12}$.

Foi estipulado um prazo de duas semanas para a devolução. Após a entrega destes questionários respondidos foram entregues aos alunos, com o mesmo prazo de duas semanas, o segundo questionário ${ }^{13}$, que avalia o cronotipo do indivíduo.

Todos os dados coletados foram inseridos em banco de dados em programa de computador para análise. Foram realizadas análises estatísticas, em forma de proporções, estabelecendo a frequência das variáveis.

\section{RESULTADOS E DISCUSSÃO}

Dos 31 acadêmicos, apenas 18 devolveram o questionário de cronobiologia totalmente respondidos, o que equivale a 58,06\%. Para este questionário foram atribuídas pontuações, classificando-os em cinco categorias comportamentais: definidamente matutinos, moderadamente matutinos, indiferentes, moderadamente vespertinos e definidamente vespertinos, com os indivíduos matutino e vespertino sendo considerados extremos cronotipos.

Para aferir o cronotipo de um indivíduo, Horne e Ostberg ${ }^{13}$ propuseram escores que variam de 16 a 86, classificados de acordo com o seguinte escore: 16 a 33, para indivíduos vespertinos; 34 a 44, para vespertinos moderados; 45 a 65 , para indiferentes; 66 a 76, para matutinos moderados; 77 a 86, para matutinos. Verificamos, em nosso estudo, um padrão de vespertinidade para os estudantes como demonstrado na Tabela1.
Tabela 1. Distribuição dos cronotipos dos alunos do primeiro ano de Medicina de uma Universidade do norte do Paraná no ano de 2016, segundo o Questionário de Identificação de Cronotipos $^{13}$

\begin{tabular}{lcc}
\hline \multicolumn{1}{c}{$\begin{array}{c}\text { Padrão de } \\
\text { cronotipo }\end{array}$} & Pontuação & $\begin{array}{c}\text { Frequência } \\
\text { relacionada ao } \\
\text { cronotipo }\end{array}$ \\
\hline $\begin{array}{l}\text { Vespertino } \\
\text { Moderadamente } \\
\text { vespertino } \\
\text { Indiferente }\end{array}$ & 36 a 33 a 41 & 8 \\
\hline Total & 45 a 52 & 6 \\
\hline
\end{tabular}

A amostra, para o questionário de Pittsburgh (PSQI), foi composta por 31 acadêmicos. Todos entregaram os questionários respondidos.

Na Tabela 2, observou-se que sete (22,58\%) alunos apresentaram boa qualidade de sono, 19 apresentaram PSQI entre 5 e 10 (61,29\%) e cinco apresentaram PSQI > 5 (16,12\%), indicando distúrbios de sono.

Tabela 2. Distribuição da qualidade do sono de 31 alunos do primeiro ano de medicina da Universidade Estadual de Maringá, segundo o Índice de Qualidade do Sono de Pittsburgh (PSQI)

\begin{tabular}{lc}
\hline Qualidade do sono & $\begin{array}{c}\text { Frequências relacionadas à } \\
\text { quantidade do sono (\%) }\end{array}$ \\
\hline Boa & $7(22,58)$ \\
Ruim & $19(61,29)$ \\
Distúrbios de sono & $5(16,12)$ \\
\hline \multicolumn{1}{c}{ Total } & $\mathbf{3 1}$ \\
\hline
\end{tabular}

Observou-se ainda que dos 19 acadêmicos que apresentaram qualidade ruim de sono, oito deles tiveram score final para o PSQI dentro do intervalo de 8 a 10 pontos, indicando que $42 \%$ estão propensos a apresentarem distúrbios de sono.

\section{DISCUSSÃO}

Os resultados evidenciaram predomínio de padrões de vespertinidade entre os acadêmicos avaliados. Sabe-se que na adolescência ocorre arrastamento do ciclo sono-vigília, o que os predispõem a dormirem e 
levantarem mais tarde, ocasionando, naturalmente, uma dessincronização com o tempo social. Nesse estudo, podese acrescentar como mais um fator de desalinhamento, a extensa grade curricular do curso de medicina que os ocupam em período integral. A ruptura da organização temporal provoca disfunções fisiológicas, promovendo suscetibilidade a diversas doenças mentais e físicas ${ }^{14}$.

O conceito de matutinidade e vespertinidade mostra inter-relação com parâmetros fisiológicos como concentração de cortisol, melatonina, temperatura corporal e ciclo sono-vigília ${ }^{15}$, todos relacionados à capacidade em se manter a atenção, o sentimento de gratificação de curto prazo e formação de memória.

Alguns autores corroboram esses dados ao mostrarem que estudantes universitários que acordam facilmente pela manhã, mostrando-se ativos nos primeiros horários do dia encontram melhores resultados acadêmicos do que os vespertinos. Além disso, os vespertinos apresentam pobre qualidade de sono, quando comparados aos matutinos ${ }^{16,17}$.

Por outro lado, apesar da preferência circadiana apresentar padrões genéticos, sofre interferências sociais, culturais e de desenvolvimento o que nos leva a inferir que ações educativas que levem esses estudantes a compreenderem a necessidade de inserirem em suas rotinas hábitos como, não levar celular ou notebook para a cama, manter o ambiente o mais escuro possível, evitar cafeína e exercícios físicos no período da noite e, aos fins de semana, expor-se à luz solar pela manhã, evitando a luz solar de fim de tarde podem contribuir para o ajuste dos ritmos biológicos.

Além disso, intervenções psicopedagógicas destinadas a promover a melhora da aprendizagem devem ser propostas, mas antes, faz-se necessário conhecer, ou levar em consideração, o perfil de cada estudante, quanto ao número de horas de sono necessárias e momento do dia em que ele melhor atua. Os ritmos circadianos e o sono, portanto, devem começar a integrar os processos de diagnósticos psicopedagógicos, dentre aqueles que são os mais comuns entre os acadêmicos ${ }^{1}$.

A análise dos dados do questionário PSQI demonstrou que 61,29\% $(n=19)$ dos acadêmicos têm sono de qualidade ruim, independentemente do cronotipo. Além disso, 42\% deles apresentaram valores de PSQI entre 8 e 10 pontos, evidenciando propensão ao desenvolvimento de distúrbios de sono.

Estudo com acadêmicos de medicina da Universidade Federal de Goiás encontrou que, dos 44 estudantes do primeiro ano, 6,8\% apresentaram sono ruim. Outro dado interessante foi o deslocamento para a direita no gráfico da amostra total da pesquisa, em que se verificou que dos 42 residentes, $21,4 \%$ encontram-se dentro do score de qualidade de sono ruim ${ }^{9}$.

Outro estudo utilizando o PSQI em alunos do curso de medicina da UFAC/AL, no primeiro semestre do ano de 2012, verificou que, dos 22 alunos do primeiro período, $54,5 \%{ }^{12}$ mostraram-se com qualidade ruim e $22,7 \%^{5}$ com distúrbio do sono ${ }^{1}$.

A sonolência excessiva diurna, mostra-se como um dos primeiros indicadores de privação de sono, podendo ser resultado de patologias do sono como distúrbios respiratórios do sono, narcolepsia e insônia, ou à insônia primária, relacionada, sobretudo a fatores sociais, profissionais, ambientais e estilo de vida, podendo a última ser revertida com mudanças de comportamento e adoção de medidas de higiene do sono.

Quando o tempo de sono é limitado por obrigações sociais e de trabalho/estudo um aumento subsequente na frequência dos episódios de sono, na duração e intensidade são verificados ao longo do dia. $\mathrm{O}$ débito de sono acumulado leva à cronicidade, que por sua vez, leva à instabilidade de atenção, diminuição do estado de alerta, memória para o trabalho prejudicada e velocidade de reação diminuída ${ }^{18}$, em algumas pessoas a insônia pode ser o desencadeante de uma futura doença mental ${ }^{19}$.

Estudantes universitários, quando recémadmitidos nas universidades ficam expostos às demandas acadêmicas e, em virtude da idade, a uma vida social mais agitada, alterando o padrão do ciclo sono-vigília na tentativa de desempenharem as atividades acadêmicas e sociais, levando-os à privação crônica de sono ao longo da vida universitária. Some-se a isso a perda da qualidade de vida, com ganho de peso, quadros hipertensivos e obesidade.

Estudo que avalia as relações privação de sono/ obesidade indica que as evidências que apoiam esta ligação nas populações adolescentes está se acumulando e que o sono deve ser otimizado em adolescentes para 
garantir resultados positivos para a saúde, contudo, acrescenta que a literatura atual para a eficácia dos programas de educação para melhorar o sono é provisória e os resultados são inconsistentes ${ }^{6}$.

\section{CONCLUSÃO}

Os resultados indicam que a população estudada é vulnerável à privação de sono, ao desenvolvimento de insônia primária e transtornos dos ritmos circadianos com consequências múltiplas e diversas.

Parece evidente, portanto que intervenções psicopedagógicas devem se concentrar na sensibilização desses estudantes sobre as consequências da privação de sono, não apenas para o desempenho acadêmico, mas sobretudo, para a qualidade de vida e longevidade, oferecendo aos alunos habilidades alternativas para lidar com as responsabilidades acadêmicas e com as novas demandas do estilo de vida da contemporaneidade.

Entende-se que essas estratégias são fáceis de implementar e podem contribuir para otimizar a qualidade do sono, mantendo os ritmos circadianos sincronizados, favorecendo o estado de alerta diurno, elevando o humor e melhorando o desempenho acadêmico.

Todavia, ações educativas para o autocuidado devem ser regulares para que todos possam, por meio da repetição, inserirem em suas rotinas os hábitos de higiene do sono, já que os resultados encontrados podem também estar relacionados ao uso do computador no horário noturno para assuntos relacionados não apenas à universidade, como as redes sociais e jogos eletrônicos.

\section{REFERÊNCIAS}

1. Faría Ribeiro, Cairon Rodrigo ; Prata da Silva, Yasmin Maria Garcia ; Carvalho de Oliveira SM. O impacto da qualidade do sono na formação médica. Rev Soc Bras Clínica Médica. 2014;55(68):8-14.

2. McCormick DA, Westbrook GL. Sono e sonhos. In: Princípios de Neurociências. 5. ed. Porto Alegre: Artmed; 2014. p. 991.

3. Figuerola WB, Ribeiro S. Sono e plasticidade neural.
2013;17-30.

4. Guissoni Campos LM, Hataka A, Vieira IZ, Buchaim RL, Robalinho IF, Arantes GEPS, et al. Circadian Clock Proteins and Melatonin Receptors in Neurons and Glia of the Sapajus apella Cerebellum. Front Physiol [Internet]. 2018;9(February):5. Available at: http://journal.frontiersin.org/article/10.3389/ fphys.2018.00005/full

5. Tan E, Scott EM. Circadian rhythms, insulin action, and glucose homeostasis. Curr Opin Clin Nutr Metab Care. 2014;17(4):343-8.

6. Arora T, Taheri S. Is sleep education an effective tool for sleep improvement and minimizing metabolic disturbance and obesity in adolescents? Sleep Med Rev [Internet]. 2017;36:3-12. Available at: https://doi. org/10.1016/j.smrv.2016.08.004

7. Marques N, Menna-Barreto L. Cronobiologia: Princípios e aplicações. 3. ed. São Paulo: Universidade de São Paulo; 2003. 435 p.

8. Lima ÁM, Dutra JRM, Tolentino Junior JC, Cortez AF, Passos RBF, Figueiredo WM de. Correlação entre Distúrbios do Sono e Avaliação Cognitiva em Estudantes de Medicina. Iniciação Científica Cesumar [Internet]. 2016;18(1):5. Available at: http:// periodicos.unicesumar.edu.br/index.php/iccesumar/ article/view/4546

9. Chagas Cardoso H, Bueno FCC, da Mata JC, Rodrigues Alves AP, Jochims I, Runulfo Vaz Filho IH, Hanna MM. Avaliação da qualidade do sono em estudantes de Medicina. Rev Bras Educ Med [Internet]. 2009;33(3):349-55. Available at: http://penelope. uchicago.edu/Thayer/E/Roman/Texts/Celsus/home. html

10. Short MA, Gradisar M, Lack LC, Wright HR. The impact of sleep on adolescent depressed mood, alertness and academic performance. J Adolesc [Internet]. 2013;36(6):1025-33. Available at: http:// dx.doi.org/10.1016/j.adolescence.2013.08.007

11. Lopes FCH. Stresse académico, afecto e qualidade do sono. Faculdade de Medicina da Universidade de 
Coimbra; 2015.

12. Buysse DJ, Reynolds CF, Monk TH, Berman SR, Kupfer DJ, III CFR, et al. The Pittsburgh Sleep Quality Index: a new instrument for psychiatric practice and research. Psychiatry Res [Internet]. 1989;28(2):193213. Available at: http://eutils.ncbi.nlm.nih.gov/entrez/eutils/elink.fcgi?dbfrom $=$ pubmed\%7B\&\%7Di$\mathrm{d}=2748771 \% 7 \mathrm{~B} \& \% 7 \mathrm{Dretmode}=\mathrm{ref} \% 7 \mathrm{~B} \& \% 7 \mathrm{Dcm}$ $\mathrm{d}=$ prlinks

13. HorneJA, OstebergO.Aself-assessementquestionnaire to determine morningness-eveningness in human circadian rhythms. Int J Chronobiol. 1976;4(2):97110.

14. Summa KC, Turek FW. Dessincronia circadiana e saúde. In: Atlas clínico de medicina do sono. Rio de Janeiro: Elsevier; 2015. p. 138.

15. Adam A, Archer SN, Hidalgo MP, Milia L Di, Vicenzo N, Christoph R. Circadian typology: A comprehensive review. Chronobiol Int. 2012;29(9):1153-75.

16. Önder I, Beşoluk Ş, Iskender M, Masal E, Demirhan E. Circadian Preferences, Sleep Quality and Sleep Patterns, Personality, Academic Motivation and Academic Achievement of university students. Learn Individ Differ. 2014;32:184-92.

17. Vollmer C, Pötsch F, Randler C. Morningness is associated with better gradings and higher attention in class. Learn Individ Differ [Internet]. 2013;27:16773. Available at: http://dx.doi.org/10.1016/j. lindif.2013.09.001

18. Spaeth AM, Abe T, Schneiderman JS. Restrição de sono. In: Atlas clínico de medicina do sono. Rio de Janeiro: Elsevier; 2015. p. 96.

19. McCall C, Shapiro CM, McCall WV. Sono e doença psiquiátrica. In: Atlas clínico de medicina do sono. Rio de Janeiro: Elsevier; 2015. p. 362.

Recebido em: 23/01/2018

Aceito em: 25/06/2018 